\title{
A narrative review of non-technical skills simulations for junior surgical trainees in managing surgical on-call
}

\section{Rebecca Smith*}

Faculty of Life Sciences and Medicine, King's College London, UK

${ }^{*}$ Corresponding author at: Faculty of Life Sciences and Medicine, King’s College London, UK. Email: rebecca.smith64@nhs.net

Date accepted for publication: 9 September 2021

\section{Abstract}

Mastery of non-technical skills (NTS) training is an essential part of surgical training but is often overlooked in favour of technical skills training. Although NTS simulations exist for several clinical activities for surgeons at all levels of training, this article identifies that there are currently no NTS simulations for junior surgical trainees in managing the surgical on-call, which is a key role assumed by senior surgeons but neglected by formal training. Development of an NTS simulation in managing the on-call would teach trainees the social, cognitive and personal resource skills that are invaluable in everyday clinical practice, improving interprofessional working relationships and overall patient care. Important NTS simulation design aspects, including the establishment of psychological safety, the impact of high sociological and psychological fidelity, and appropriate simulation evaluation methods are discussed in depth. A ready-to-use template for a potential pilot study is included for further research into developing a low-cost, highimpact NTS simulation concept into future surgical training.

Keywords: non-technical skills; simulation; surgical training; surgical trainees; junior surgical trainees; managing on-call

\section{Introduction}

Surgical training has evolved substantially in recent years; no longer is it acceptable to rely on technical proficiency alone; there is an expectation for trainees to develop mastery in non-technical skills (NTS) also. ${ }^{1-4}$ NTS can be categorized into social (teamwork, leadership, communication), cognitive (decision-making, situational awareness) and personal resource factors (ability to cope with stress and fatigue). ${ }^{5}$ These skills not only facilitate cohesive interprofessional relationships within institutions but also affect clinical and patient outcomes. Communication breakdown, poor decision-making and inadequate team-working have been shown to result in increased errors and threaten patient safety. ${ }^{3,6-8}$ Contemporary literature supports an increasing evidence base for developing NTS in future surgeons, influencing a drive for NTS training to be incorporated into modern surgical curriculum design. ${ }^{9-12}$

Core surgical trainees (CSTs) in the United Kingdom are junior surgical trainees who undertake 2 years of general and sub-specialty surgical rotations before applying for specialized higher surgical training as a registrar. Workload is divided between operating, ward rounds, outpatient clinics, administrative work and teaching. ${ }^{12}$ A proportion of the surgical rota is dedicated to being on-call, and during a standard day shift, there is one foundation doctor, one senior house officer and one registrar each carrying an on-call bleep. Typically, the on-call shift is extremely busy, involving but not limited to the following areas: taking referrals from other specialties within the hospital or from primary care; clerking and admitting new patients; reviewing ambulatory care patients; managing acutely unwell inpatients; performing emergency procedures and operations. These various tasks are delegated across the on-call team depending on surgical experience, with higher training grades generally receiving calls about the more complex cases. Managing the on-call tests not only a doctor's clinical knowledge and technical ability but also NTS and professional behaviours. ${ }^{11,12}$

\section{Aims and methodology}

This is a narrative review of the literature, which aims to identify the nature and type of NTS training available for junior surgical trainees in managing the on-call, or to facilitate the transition from junior to senior surgical trainee (i.e. CST to registrar) in a non-operative setting. The secondary aim is to examine the extent to which research is published looking at types of clinical NTS training, method of delivery and target audience for NTS training. 
A comprehensive literature search of the online database PubMed was performed using the following search terms for all articles published before April 2021:

"Non-technical skills surgical trainees"

"Non-technical skills surgical training"

"Non-technical skills junior surgical trainees"

"Non-technical skills managing on-call"

"Non-technical skills managing bleep"

"Non-technical skills managing pager"

"Non-technical skills simulation"

"Non-technical skills simulation surgical training"

"Non-technical skills surgical registrar"

"Non-technical skills core surgical training"

\section{Results}

Abstracts were screened for relevance by the author. The reference lists of relevant papers were subsequently screened for additional cross-referenced articles. In total, 13 relevant articles with a focus on NTS simulation for surgical trainees were used as core resource material.

There were no articles identified that specifically focused on NTS training for junior surgical trainees in managing the on-call or to facilitate the transition from junior to senior surgical trainee in a non-operative setting. Of the other relevant articles identified, existing literature focuses on the following NTS training for surgical trainees:

- Clinical activities: (1) ward round simulations; ${ }^{13-15}$ operating room simulations; ${ }^{10,16,17}$ (3) multidisciplinary team meetings. ${ }^{18}$

- Method of NTS delivery: (1) didactic courses; ${ }^{8,19}$ (2) human factors training; ${ }^{20}$ (3) NTS training alongside technical skills simulation. ${ }^{4,16,19}$

- Target audience: (1) medical students; ${ }^{21}$ (2) junior surgical trainees; ${ }^{10,15,20}$ (3) senior trainees or consultant surgeons. ${ }^{8,9,16}$

\section{Discussion and narrative review}

A review of the literature in PubMed did not identify any articles that specifically focused on NTS training for junior surgical trainees in managing the on-call or facilitated the transition from junior to senior surgical trainee in a nonoperative setting.
For the secondary aim, a literature review identified a number of papers focusing on various types of clinical NTS training, method of delivery and target audience for NTS training, as summarized in the results. The aim of this review was not to perform any analysis of this literature, therefore no data are presented for the secondary aim. This form of analysis remains a strategy for future research in the form of a systematic review of the literature, which the author is currently undertaking.

In the absence of specific relevant data in the literature, the author proposes a research template for a pilot study focusing on NTS training for CSTs in managing the on-call to facilitate the transition from CST to registrar in a nonoperative setting. This is presented after a discussion of the rationale for NTS simulations for junior surgical trainees and analysis of design considerations for a simulation concept.

\section{Rationale for NTS simulations for junior surgical trainees}

In addition to improved patient safety, the following arguments justify the development of a simulation-based intervention to improve NTS in junior surgical trainees.

\section{Surgical curriculum}

Evidence of good NTS and professionalism is required as part of the Intercollegiate Surgical Curriculum Programme. ${ }^{11}$ Whereas knowledge and skills are explicitly trained and assessed in the form of postgraduate exams and work-based assessments, it is often left to the "hidden curriculum' to address developing trainee professionalism. ${ }^{12}$ In addition, the Intercollegiate Surgical Curriculum Programme (ISCP) standards of training state that 'surgeons need to be able to perform in differing conditions and circumstances, respond to the unpredictable, and make decisions under pressure, frequently in the absence of all the desirable data ... use professional judgement, insight and leadership in everyday practice, working within multiprofessional teams. ${ }^{11}$ An on-call simulation focusing on NTS combines these standards with a safe environment, potentiating valuable learning gains while removing all risk of patient harm.

\section{Pushing boundaries of competence}

Although established NTS simulations exist, such as the Non-Technical Skills for Surgeons (NOTSS) course run by the Royal College of Surgeons of Edinburgh, ${ }^{22}$ these are targeted towards more senior trainees and consultants. This leaves a subgroup of junior surgeons somewhat neglected, despite arguably presenting the greatest need for such formative training. ${ }^{16}$ Transitioning up the training grade ladder carries weightier responsibilities and 
autonomy, which many may find daunting; although this is largely anecdotal, Fry and Kneebone ${ }^{1}$ accurately described how the transition from CST to registrar can take some trainees by surprise: 'it's when someone junior rings you up for advice - that doesn't happen to you when you're junior. One day you're phoning for advice and the next you're the one being phoned and that's an external thing that's imposed upon you.' ${ }^{\prime 1}$ Although the step up from registrar to consultant is acknowledged through established guidance for 'acting up' as a consultant, no such framework exists for CST to registrar. ${ }^{11}$ In addition, most of the established NTS simulations involve scenarios within the operating room, ${ }^{23}$ despite evidence that trainees spend most of the working week outside of theatre. ${ }^{24}$

\section{Abundance of technical skills simulations}

The traditional focus on operative skills as the defining attribute of good surgeons appears to have infiltrated the agenda of simulation-based training; the quantity and availability of simulations disproportionately favours technical over NTS training, despite the recognized importance of the latter. ${ }^{3}$

\section{Design considerations for a potential NTS simulation}

There are several design aspects that could be discussed in depth in the conception of an appropriate NTS simulation for managing an on-call and transitioning to a more senior surgical role. Three particular areas are discussed here based on maximizing the usefulness of the intervention to the target audience and key simulation-specific considerations documented in the existing literature.

\section{Psychological safety and its role in debriefing}

Psychological safety (PS) is described as 'people's perceptions of the consequences of taking interpersonal risks in a particular context ${ }^{25}$ existing within three levels: the individual, the group and the organization. ${ }^{26}$ This can be applied to this particular context as a CST's willingness to attempt new practices and risk vulnerability for the sake of learning (the individual), the level of openness, trust and respect among participating colleagues and faculty (the group), and the wider support afforded by the surgical department (the organization). A high degree of PS is essential in this simulation; not only because effective debriefing depends on its presence ${ }^{26}$ but also because its absence could result in detrimental effects to individual learning, interpersonal relationships and future practice and behaviours. This is perhaps better explained with an example. A performance-oriented CST participating in a simulation with perceived low PS will strive to seek good judgement from peers and faculty, leading them to steer the simulation down a fairly well-trodden learning path for the perceived benefit of portraying themselves as an excellent trainee. The same CST may also feel uncomfortable opening up about any concerns or anxieties they felt during the simulation debriefing for fear of ridicule and ill judgement. This occurs at the expense of minimal individual, group and faculty learning gains, negligible behavioural change, and possible cultivation of poor group learning behaviours such as defensiveness, disengagement, and disingenuousness. It is therefore imperative that the proposed simulation fosters an environment where candidates feel safe about making mistakes and welcome criticism to improve their own knowledge, skills and behaviours in future practice.

One could perhaps analogize the management of PS in simulation to primary, secondary and tertiary prevention of disease, where primary prevention is PS establishment, secondary prevention is PS maintenance, and tertiary prevention is restoration of PS following breaches, the intention being that sufficient PS establishment should avoid development of PS breaches altogether, and thus optimal PS management is enabled by careful consideration of PS establishment in scenario design. It can be helpful to think of desirable simulation design as a 'safe container', a metaphor described by Rudolph et al. $^{27}$ as 'a context where difficult conversations, emotions or potentially threatening feedback can be tolerated and transformed into generative material in the learning process.' However, the authors recognized that it is not enough to simply announce the presence of a safe space, but instead one must take positive actions surrounding simulation design and implementation towards PS establishment. Details of strategies that might contribute to PS before, during and after debriefing are discussed in depth by Kolbe et al. ${ }^{26}$ and include the following.

- Implicit strategies: arriving early, creating a private environment, circular seating, showing empathy, eye contact, pausing to listen, behavioural integrity, being mindful of timing

- Explicit strategies: clarifying expectations, assuring confidentiality, transparency, inclusivity, paraphrasing, authenticity, normalization, vulnerability, showing curiosity and appreciation, offering support, inviting feedback

PS is crucial for effective debriefing. Some strategies, such as arriving early and offering support, are fairly straightforward actions that can be easily implemented by a facilitator 
regardless of experience. Others, such as paraphrasing, authenticity and normalization, are far more dependent on debriefer skill. This is an area of concern; without appropriate faculty experience, there is a risk to PS establishment and maintenance. However, without knowing who exactly the faculty will be, any concerns are indeed valid but speculative. To minimize reliance on faculty experience, faculty training would be essential to set out the goals and expectations of debriefing, the culture in which it will be implemented, and the strategies to facilitate a psychologically safe environment. There are a number of debriefing styles that would support PS while maximizing learning, such as advocacy-enquiry ${ }^{28}$ and 'The Diamond' debrief. ${ }^{29}$ Debriefing is a hugely complex topic deserving of a detailed discussion in itself and is thus beyond the scope of this article. However, it is necessary for faculty to interrogate their intended debriefing practice and how this will establish and preserve PS in NTS simulations.

\section{Fidelity: sociological and psychological}

Interprofessional conflicts and hierarchies are ubiquitous in medicine, and surgical specialties are certainly no exception to this. An objective of this simulation is to improve interprofessional collaboration by exposing and addressing areas of sociological conflict within surgical practice and the wider health care context in which surgery operates. Sharma et al. ${ }^{30}$ argued that although individual skill acquisition is important, this is too narrow a focus of education; if professionals are to work collaboratively and effectively, then the intended clinical context in which these skills are to be implemented must be integrated into teaching design. This emphasizes the importance of high sociological fidelity (SF) within this simulation; the scenarios must reflect the real tensions, hierarchies and professional boundaries that exist in clinical practice.

As all candidates will be CSTs, this simulation is not explicitly interprofessional. However, candidates will be communicating with a variety of professionals via simulated phone calls. There are several options for who can 'act' as these professionals. Faculty members could act as health care professionals, however this would rely on experienced faculty knowing and replicating existing professional dynamics during simulation. Failure to realistically portray this risks $\mathrm{SF}$ and is thus a heavy burden for faculty to bear. An alternative could involve the actual professionals in simulation design, i.e. the real $\mathrm{A} \& \mathrm{E}$ registrar calls to make a referral, rather than a role player, facilitating a transition from uni-professional to multi-professional simulation. Although this would undoubtedly improve SF and contribute to richer debrief discussion, this solution poses far greater logistical problems by having to coordinate the schedules of numerous faculty from various specialties for the simulation to run smoothly. This aspect of simulation design will need to be trialled and refined in the early stages of simulation before finding a solution that works and is justified by evaluative methods. Nevertheless, a focus on high SF in simulation will facilitate meaningful and relevant learning experiences for CSTs.

Psychological fidelity (PF), the degree to which a CST finds this scenario to be believable, is also an important aspect of scenario design and can improve information recall and application, ${ }^{31}$ The simulation should reflect the real-life pressures that surgical registrars experience on a busy oncall. One particular concern is that candidates may not feel obliged to make difficult decisions knowing that the simulation will end and their actions will have no real-life consequences. DeMaria et al. ${ }^{32}$ explored how the addition of emotional stressors can be more realistic. In this context, PF could be manipulated by piling more pressure on candidates than would likely be the case in real practice, for example, by increasing the frequency of bleeps or by setting conditions on how many referrals can be accepted due to a limited number of beds available. Although a high degree of PF is desirable, it is important to note its symbiotic existence alongside PS; if simulation PS is sufficient, candidates will feel more comfortable in an increasingly pressurized environment, allowing incorporation of additional stressors to result in a higher PF simulation. This emphasizes the relationship of individual design aspects with one another, and how simulation design must be considered as a whole rather than separate entities.

\section{Evaluation}

The Kirkpatrick Model ${ }^{33}$ can be used to evaluate the effects of simulation, with learning occurring on four levels:

- Level 1: reaction. 'The degree to which participants find the training favourable, engaging and relevant to their jobs', i.e. are candidates satisfied?

- Level 2: learning. 'The degree to which participants acquire the intended knowledge skills, attitude, confidence and commitment based on their participation in training', i.e. is there evidence of improved skill?

- Level 3: behaviour. 'The degree to which participants will apply what they have learned during training when they are back on the job', i.e. are these skills being used in practice? 
- Level 4: results. 'The degree to which targeted outcomes occur as a result of the training and the support and accountability package', i.e. is this leading to improved patient outcomes? ${ }^{34}$

Evidence of level 4 learning provides the strongest justification for the effectiveness of simulation but is the most difficult to evaluate in practice. ${ }^{35}$ It seems near impossible to devise a method in which improved NTSs in a small subset of trainees can be causatively and definitively linked with improved patient care. Perhaps a solution lies in evaluating behaviours as the next desirable effect of learning, by gathering qualitative data over time from CSTs and those that they interact with to determine whether learnt skills have been applied in practice. This leads to another dilemma. How far can researchers rely on this qualitative data if they are self-reported (i.e. from CST feedback) or selfselected (i.e. from multi-source feedback), and how well do self-reported outcomes translate into improved clinical practice? Not only are there concerns that knowledge tests and self-confidence measures do not predict clinical performance, ${ }^{36}$ but there are also many limitations of self-reported questionnaires as a valid evaluation tool. ${ }^{37}$ Finally, how do researchers prove that the simulation actually works compared with no simulation intervention? Establishing a control group for comparison would be difficult here. It is fairly unrealistic that a group of keen CSTs selected as a control group, expecting equal training opportunities from their surgical training programmes, would accept the notion that their colleagues will receive simulation training and they will not.

No evaluation method will be perfect. However, during the early conception of this simulation, the gathering of rich qualitative data from all stakeholders will serve to refine and reshape the simulation to address the needs and expectations of CSTs and the wider organization in which they operate. Pre- and post-intervention surveys can collect quantitative data assessing self-reported confidence in the three areas of NTS (social, cognitive and personal resource factors), with a comments section to encourage trainees to provide further qualitative details. Although confidence does not necessarily equate to good surgical performance, it is recognized that a high degree of confidence supports trainees in a challenging and competitive field. ${ }^{15}$ In addition, qualitative longitudinal data can be collected from CSTs over time using interviews/questionnaires and multi-source feedback to demonstrate how, as a cohort, application of NTSs in practice has improved over the training period. The intention is to incorporate different data types, at different time points, and from different sources, to provide a broad dataset from which to finesse the simulation over time; admittedly this is a fairly wide net to cast, but its purpose is to provide a richness in data that will improve the effectiveness of this simulation in achieving its learning objectives.

\section{Template for a potential pilot study}

Appendices 1-3 provide a ready-to-use template for a potential pilot study in developing an NTS simulation for CSTs in managing the on-call.

(1) Simulation set-up. This gives instructions to faculty and candidates about what the scenario intends to achieve, what equipment and resources are required and how the simulation should run.

(2) Scenario. One example scenario is provided, which is intentionally designed to generate a wide breadth of different possible outcomes depending on candidate response, leading to rich and varied discussion points during the debrief. Ideally, different iterations of the scenario will generate different learning points because there is no one prescribed or 'correct' route that the candidate should follow. It is important to focus on NTS rather than technical skills or clinical knowledge.

(3) Survey. Using pre- and post-intervention surveys, quantitative data can be collected assessing selfreported confidence in the three areas of NTS (social, cognitive and personal resource factors), with a comments section to encourage trainees to provide further qualitative details.

\section{Limitations}

The literature search was carried out by a single author using PubMed as the only search engine, and it is possible some grey literature or published papers would have been found if more search engines were used to search this topic. No data could be presented due to complete paucity of literature on the topic of NTS training for junior surgical trainees in managing the on-call or to facilitate the transition from junior to senior surgical trainee in a non-operative setting.

\section{Conclusion}

Mastery of non-technical skills is an essential part of surgical training but is often overlooked in favour of technical 
skills training. Core surgical trainees are expected to acquire the necessary non-technical skills to become successful registrars, despite a paucity of explicit non-technical skills training. This article outlines the themes of existing NTS simulations for surgical trainees, highlighting the absence of NTS training for CSTs in the management of the surgical on-call. A simulation-based intervention could facilitate CSTs to not only navigate the daunting transition to surgical registrar but also achieve the social, cognitive and personal resource skills that are invaluable in everyday clinical practice, improving interprofessional working relationships and overall patient care. Maximal effectiveness of NTS simulation relies on careful consideration of certain design aspects, such as the establishment of psychological safety, the impact of high sociological and psychological fidelity, and appropriate simulation evaluation methods. The next step for future research would be to conduct a pilot study, perhaps using the template presented in this article, to evaluate the effectiveness, acceptability and feasibility of a novel simulation-based intervention.

\section{Acknowledgements}

Thank you to Dr Ranjev Kainth (King's College London) for his invaluable support when writing this article.

\section{Conflict of interest}

None declared.

\section{References}

1. Fry H, Kneebone R. Surgical education: theorising an emerging domain. Dordrecht: Springer; 2011. https://doi.org/10. 1007/978-94-007-1682-7.

2. Hurreiz $H$. The evolution of surgical training in the UK. Adv Med Educ Pract 2019; 10: 163-168. https://doi.org/10. 2147/AMEP.S189298.

3. Agha RA, Fowler AJ. The role and validity of surgical simulation. Int Surg 2015; 100(2): 350-357. https://doi.org/10. 9738/INTSURG-D-14-00004.1.

4. Lynch A. Simulation-based acquisition of non-technical skills to improve patient safety. Semin Pediatr Surg 2020; 29(2): 150906. https://doi.org/10.1016/j.sempedsurg.2020.150906.

5. Flin R, O'Connor P, Crichton M. Safety at the sharp end: a guide to non-technical skills. Burlington, VT: Ashgate; 2008.

6. Gawande AA, Zinner MJ, Studdert DM, Brennan TA. Analysis of errors reported by surgeons at three teaching hospitals. Surgery 2003; 133(6): 614-621. https://doi.org/10. 1067/msy.2003.169.
7. Rogers SO Jr, Gawande AA, Kwaan M, Puopolo AL, Yoon C, Brennan TA, et al. Analysis of surgical errors in closed malpractice claims at 4 liability insurers. Surgery 2006; 140(1): 25-33. https://doi.org/10.1016/j.surg.2006.01.008.

8. Gostlow H, Marlow N, Thomas MJ, Hewett PJ, Kiermeier A, Babidge W, et al. Non-technical skills of surgical trainees and experienced surgeons. Br J Surg 2017; 104(6): 777-785. https://doi.org/10.1002/bjs.10493.

9. Ounounou E, Aydin A, Brunckhorst O, Khan MS, Dasgupta $\mathrm{P}$, Ahmed K. Nontechnical skills in surgery: a systematic review of current training modalities. J Surg Educ 2019; 76(1): 14-24. https://doi.org/10.1016/j.jsurg.2018.05.017.

10. Dedy NJ, Bonrath EM, Ahmed N, Grantcharov TP. Structured training to improve nontechnical performance of junior surgical residents in the operating room: a randomized controlled trial. Ann Surg 2016; 263(1): 43-49. https://doi.org/10. 1097/SLA.0000000000001186.

11. ISCP. The Intercollegiate Surgical Curriculum: educating the surgeons of the future. 2017. Available from: https://www.iscp. ac.uk/static/public/syllabus/syllabus_core_2017.pdf.

12. The Royal College of Surgeons of England. Improving surgical training. London: The Royal College of Surgeons of England: Professional Standards; 2015. Available from: https://www. rcseng.ac.uk/library-and-publications/rcs-publications/docs/im proving-surgical-training/.

13. Somasundram K, Spence H, Colquhoun AJ, Mcilhenny C, Biyani CS, Jain S. Simulation in urology to train non-technical skills in ward rounds. BJU Int 2018; 122(4): 705-712. https://doi.org/10.1111/bju.14402.

14. Harvey R, Mellanby E, Dearden E, Medjoub K, Edgar S. Developing non-technical ward-round skills. Clin Teach 2015; 12(5): 336-340. https://doi.org/10.1111/tct.12344.

15. Bamford R, Langdon L, Rodd CA, Eastaugh-Waring S, Coulston JE. Core trainee boot camp, a method for improving technical and non-technical skills of novice surgical trainees. A before and after study. Int J Surg 2018; 57: 60-65. https://doi.org/10.1016/j.ijsu.2018.03.083.

16. Griffin C, Ardin A, Brunckhorst O, Raison N, Khan MS, Dasgupta $\mathrm{P}$, et al. Non-technical skills: a review of training and evaluation in urology. World J Urol 2020; 38: 1653-1661. https://doi.org/10.1007/s00345-019-02920-6.

17. Robertson JM, Dias RD, Yule S, Smink DS. Operating room team training with simulation: a systematic review. J Laparoendosc Adv Surg Tech A 2017; 27(5): 475-480. https://doi.org/10.1089/lap.2017.0043.

18. Trivedi DB. Educational value of surgical multidisciplinary team meetings for learning non-technical skills - a pilot survey of trainees from two UK deaneries. J Surg Educ 2019; 76(4): 1034-1047. https://doi.org/10.1016/j.jsurg.2019. 02.001 . 
19. Wood TC, Raison N, Haldar S, Brunckhorst O, McIlhenny C, Dasgupta $\mathrm{P}$, et al. Training tools for nontechnical skills for surgeons-a systematic review. J Surg Educ 2017; 74(4): 548578. https://doi.org/10.1016/j.jsurg.2016.11.017.

20. Jones M, Howells N, Mitchell S, Burnand H, Mutimer J, Longman R. Human-factors training for surgical trainees. Clin Teach 2014; 11(3): 165-169.https://doi.org/10.1111/tct. 12147.

21. Dickinson M, Pimblett M, Hanson J, Davis M. Reflecting reality: pager simulations in undergraduate education. Clin Teach 2014; 11(6): 421-424. https://doi.org/10.1111/tct.12185.

22. The Royal College of Surgeons of Edinburgh. Non-Technical Skills for Surgeons (NOTSS). Available from: https://www. rcsed.ac.uk/media/682516/notss-system-handbook-v20.pdf.

24. BOTA Collaborators, Rashid MS. An audit of clinical training exposure amongst junior doctors working in Trauma \& Orthopaedic Surgery in 101 hospitals in the United Kingdom. BMC Med Educ 2018; 18(1): 1. https://doi.org/10. 1186/s12909-017-1038-5.

25. Edmondson AC, Lei Z. Psychological safety: the history, renaissance, and future of an interpersonal construct. Annu Rev Organ Psychol Organ Behav 2014; 1(1): 23-43. https://doi.org/10.1146/annurev-orgpsych-031413-091305.

26. Kolbe M, Eppich W, Rudolph J, Meguerdichian M, Catena H, Cripps A, et al. Managing psychological safety in debriefings: a dynamic balancing act. BMJ Simul Technol Enhanc Learn 2020; 6(3): 164-171. https://doi.org/10.1136/bmjstel-2019000470.

27. Rudolph JW, Raemer DB, Simon R. Establishing a safe container for learning in simulation: the role of the presimulation briefing. Simul Healthc 2014; 9(6): 339-349. https://doi.org/10. 1097/SIH.0000000000000047.

28. Rudolph JW, Simon R, Dufresne RL, Raemer DB. There's no such thing as "nonjudgmental" debriefing: a theory and method for debriefing with good judgment. Simul Healthc
2006; 1(1): 49-55. https://doi.org/10.1097/01266021200600110-00006.

29. Jaye P, Thomas L, Reedy G. 'The Diamond': a structure for simulation debrief. Clin Teach 2015; 12(3): 171-175. https://doi.org/10.1111/tct.12300.

30. Sharma S, Boet S, Kitto S, Reeves S. Interprofessional simulated learning: the need for 'sociological fidelity'. J Interprof Care 2011; 25(2): 81-83. https://doi.org/10.3109/13561820. 2011.556514.

31. Khan K, Pattison T, Sherwood M. Simulation in medical education. Med Teach 2011; 33(1): 1-3. https://doi.org/10.3109/ 0142159X.2010.519412.

32. Demaria S Jr, Bryson EO, Mooney TJ, Silverstein JH, Reich $\mathrm{DL}$, Bodian $\mathrm{C}$, et al. Adding emotional stressors to training in simulated cardiopulmonary arrest enhances participant performance. Med Educ 2010; 44(10): 1006-1015. https://doi.org/10. 1111/j.1365-2923.2010.03775.x.

33. Kirkpatrick D. Revisiting Kirkpatrick's four-level-model. Train Dev 1996; 50: 54-57.

34. Kirkpatrick D, Kirkpatrick J. Transferring learning to behaviour: using the four levels to improve performance. San Francisco, CA: Berrett-Koehler; 2005.

35. Kurt S. Kirkpatrick Model: four levels of learning evaluation. Educ Technol 2016. Available from: https://educationaltech nology.net/kirkpatrick-model-four-levels-learning-evaluation/.

36. Liaw SY, Scherpbier A, Rethans JJ, Klainin-Yobas P. Assessment for simulation learning outcomes: a comparison of knowledge and self-reported confidence with observed clinical performance. Nurse Educ Today 2012; 32(6): e35-e39. https://doi.org/10.1016/j.nedt.2011.10.006.

37. Demetriou C, Özer BU, Essau CA. Self-report questionnaires. In: Cautin RL, Lilienfeld SO, editor. The encyclopedia of clinical psychology. New York: John Wiley; 2015. https:/doi. org/10.1002/9781118625392.wbecp507. 


\section{Appendix 1: Simulation set-up}

\begin{tabular}{|c|c|c|}
\hline \multicolumn{3}{|c|}{ Managing a surgical on-call - transition from CST to Registrar } \\
\hline Learning Goal & \multicolumn{2}{|c|}{$\begin{array}{l}\text { 1. To learn and practice managing a busy surgical on-call } \\
\text { 2. To develop non-technical skills, including teamwork, leadership, communication, } \\
\text { decision-making, situational awareness, ability to cope with stress and fatigue. } \\
\text { 3. To demonstrate effective inter-disciplinary and interprofessional collaboration in } \\
\text { achieving common goals. }\end{array}$} \\
\hline $\begin{array}{l}\text { Narrative } \\
\text { Description }\end{array}$ & \multicolumn{2}{|c|}{$\begin{array}{l}\text { It is } 6 \mathrm{pm} \text { on a Friday and you are on-call as the general surgical registrar in a District General } \\
\text { Hospital. You are waiting in the theatre coffee room as the anaesthetist is currently intubating } \\
\text { your next case, a } 12 \text { year old girl requiring an emergency laparoscopic appendicectomy. You } \\
\text { are alone, but your SHO is due to arrive in order to assist you, and the rest of your on-call team } \\
\text { (consultant and FY1) are contactable and on site. You have your bleep, a computer, a pen and } \\
\text { paper. }\end{array}$} \\
\hline Case Briefing & \multicolumn{2}{|c|}{$\begin{array}{l}\text { While waiting for your next case, your bleep will go off. Please manage the on-call } \\
\text { appropriately, prioritising, delegating and escalating tasks as necessary. If you need to call a } \\
\text { colleague, please dial (xxxx) to speak to switchboard, who will then connect you. The scenario } \\
\text { will last } 20 \text { minutes. This will be followed by a } 20 \text { minute de-brief. }\end{array}$} \\
\hline \multirow[t]{2}{*}{ Staffing } & $\begin{array}{l}\text { Faculty: } \\
\text { A minimum of } 1 x \text { role player (ideally } \\
\text { with surgical experience) } \\
1 \times \text { facilitator/observer (no surgical } \\
\text { experience required) }\end{array}$ & $\begin{array}{l}\text { Candidates: } \\
\text { A minimum of } 1 x \text { core surgical trainee/surgical } \mathrm{SHO}\end{array}$ \\
\hline & $\begin{array}{l}\text { Faculty Control Room: } \\
\text { Room separate from candidates } \\
\text { Telephone with connection to } \\
\text { participant telephone } \\
\text { Pen and paper }\end{array}$ & $\begin{array}{l}\text { Candidates: } \\
\text { Room separate from faculty } \\
\text { Desk with computer, bleep, telephone, paper and } \\
\text { pen } \\
\text { Connection to system network (internet and } \\
\text { landline) } \\
\text { Chairs } \\
\text { Optional - whiteboard and pens to use for debrief }\end{array}$ \\
\hline Props needed & \multicolumn{2}{|c|}{ Computer, bleep, telephone, paper and pen } \\
\hline $\begin{array}{l}\text { Proposed } \\
\text { simulation } \\
\text { structure }\end{array}$ & \multicolumn{2}{|c|}{$\begin{array}{l}\text { Introductions + simulation briefing - } 10 \text { min } \\
\text { Please use this time to re-iterate the importance of psychological safety - candidates should } \\
\text { feel at ease, rather than any sense of impending judgement or consequence from either faculty } \\
\text { or other participants. } \\
\text { Scenario - } \mathbf{2 0} \text { min } \\
1 \text { candidate to volunteer for the scenario, the remaining candidates to be actively involved in } \\
\text { observation. These candidates could be assigned particular roles during observation, e.g. a } \\
\text { scribe to timeline events or decisions made in order to generate discussion points in the } \\
\text { debrief. } \\
\text { Debrief - } 20 \text { min } \\
\text { The format of the debrief is at faculty discretion, but must be where the key learning points are } \\
\text { generated. This means that even those candidates who observe the scenario can still gain } \\
\text { useful training. The focus should remain on non-technical skills, rather than clinical knowledge. } \\
\text { Recommended debriefing structures include advocacy-enquiry and the Diamond debrief. }\end{array}$} \\
\hline
\end{tabular}

\section{Notes for Faculty:}

- The scenario is intended to be pressurised, but is designed to focus on non-technical skills rather than clinical knowledge. Please prepare the scenario provided in advance, thinking about potential paths the scenario could take.

- The role player will call the candidate using the paging system and assume one the role from the scenario (i.e. the A\&E registrar).

- When calling the candidate, please keep the telephone on speaker so both faculty staff can listen to the conversation. 
- If the candidate needs to call another member of staff, they will dial 'switchboard' on xxxx, which should be the number of the faculty telephone. Please act as switchboard, before assuming the role of the requested member of staff (after a short pause, if required, to prepare yourself for this role).

- Additional stressors can be considered at the discretion of faculty according to candidate experience (e.g. additional bleeps, difficult colleagues, pressure from theatres).

- The facilitator should keep a record of the scenario, including a timeline and discussion points for the debrief, or assign this role to one of the observing candidates.

- Be flexible and reactive - the scenario will be unique depending on the candidate responses, so do try and accommodate this.

- The scenario is dynamic and can be refined and adjusted according to candidate and faculty feedback.

- New example scenarios can be added periodically in order to create a wide breadth of questions which can be reused for multiple simulations, even with the same candidate.

- Establishment and maintenance of psychological safety is essential throughout the simulation.

- Detailed feedback from candidates is strongly encouraged to provide a rich qualitative dataset for informing future simulations. 


\section{Appendix 2: Scenario: A\&E referral}

- You are the A\&E registrar. You are calling the surgical registrar to urgently review a patient who has just been brought in by ambulance with an acute abdomen:

o History: 65-year-old male previously fit and well found collapsed at home, now complaining of severe central abdominal pain

o Past medical history: hypertension, smoker, type 2 diabetic, previous cholecystectomy

- Your team has performed a rapid assessment of the patient and are currently resuscitating the patient. Based on the patient's symptoms and presentation, you are concerned that there is an underlying acute surgical pathology

o If asked, $\mathbf{A}$ to $E$ assessment includes:

$\square$ A - patent

$\neg$ B - RR 24, O2 $96 \%$ on $100 \% 02$ via non-rebreathe mask, equal breath sounds with reduced air entry bibasally on auscultation

$\square \mathrm{C}$ - patient is pale and clammy, HR 125 irregularly irregular, BP 85/65, CRT 4 seconds, has not passed urine since arriving in department

$\square$ - alert but agitated, blood glucose $15.6 \mathrm{mmol} / \mathrm{L}$, temperature $37.8^{\circ} \mathrm{C}$

$\square \mathrm{E}-$ abdomen is mildly distended, peritonitic and generally tender, no evidence of PR bleeding/melaena/haematemesis

o If asked, current management is:

$\square$ IV cannulation and fluid resuscitation

$\neg$ Bloods sent including FBC, U\&Es, CRP, LFTs, amylase, clotting, crossmatch 2 units, ABG

$\checkmark$ Plan for urinary catheter and measurement of urine output

$\square$ Portable CXR requested

$\neg$ Cardiac monitoring

$\square$ No further plans for imaging as patient is not yet stable enough

$\square$ FAST scan showed what looks like free fluid in the abdomen

- You're not entirely sure what the diagnosis but you think they definitely need an urgent surgical review

o If asked, possible differentials include:

\section{$\neg$ Ruptured AAA}

$\neg$ Acute severe pancreatitis

$\square$ Bowel perforation

$\neg$ Bowel ischaemia

o If asked, you have not yet involved any other specialties or told anyone else from the surgical team about this patient

- You are extremely concerned about the patient. You stress the importance of an urgent surgical review and you suspect that they may require an emergency laparotomy. There are many possible responses the candidate may provide, for example:

o If the candidate asks you to call the SHO, you can accept this reluctantly, although stress that you think this patient really needs have a senior review due to the severity of their presentation.

o If the candidate asks you to call the consultant, you could be hesitant about this and ask the candidate to speak to the consultant themselves, but if sufficient explanation is provided then you can also accept this. However, you should not allow the candidate to relinquish responsibility of the patient.

o If the candidate does not delegate or says they are too busy, you can say that this is unacceptable and ask for immediate review.

Following this referral, the candidate may decide to then call a colleague, e.g. SHO, consultant, to ask for advice/ask colleague to review patient/ask colleague to come to theatre to take over appendicectomy, etc.

Please feel free to improvise depending on candidate response. Example responses from colleagues could include:

- Consultant

o Sounds like a ruptured AAA - you should ring the vascular team

o Sounds like a perforated viscus - you should get theatres ready

o Sounds like severe pancreatitis - you should speak to ITU

o I could go down to A\&E and see the patient, but what's the diagnosis? Why hasn't a CT happened?

o You could quickly go to A\&E now as the anaesthetist is still intubating and they could wait for you to come back

o I could come and take over the appendicectomy, but l'll be half an hour

- $\mathrm{SHO}$

o I'm just on my way to join you in theatres now - what should I do?

o I could go see them but I don't feel hugely confident in managing someone who sounds so unwell

o I'm caught up on the ward with the F1 as a patient has acutely deteriorated post-op

- Anaesthetist

o Your patient is ready - are you going to operate or are you getting someone else?

o I don't think it's appropriate for you to go to A\&E and leave this paediatric patient on the table

o l'd have to get a colleague to assess this new patient in A\&E, or else they'll have to wait until after the

- Theatre staff appendicectomy

o There's only got emergency theatre available, which we are doing the appendicectomy in. We'll have to call in more staff if you want to open another one

o What equipment do you need? Who will do the emergency case in A\&E? Who will do the appendicectomy? 


\section{Appendix 3: Survey}

Pre- and post-intervention survey for candidates, with responses scored using a five-point Likert scale (candidates scoring 5 if strongly agree and 1 if strongly disagree). Based on NOTSS questionnaire. ${ }^{22}$

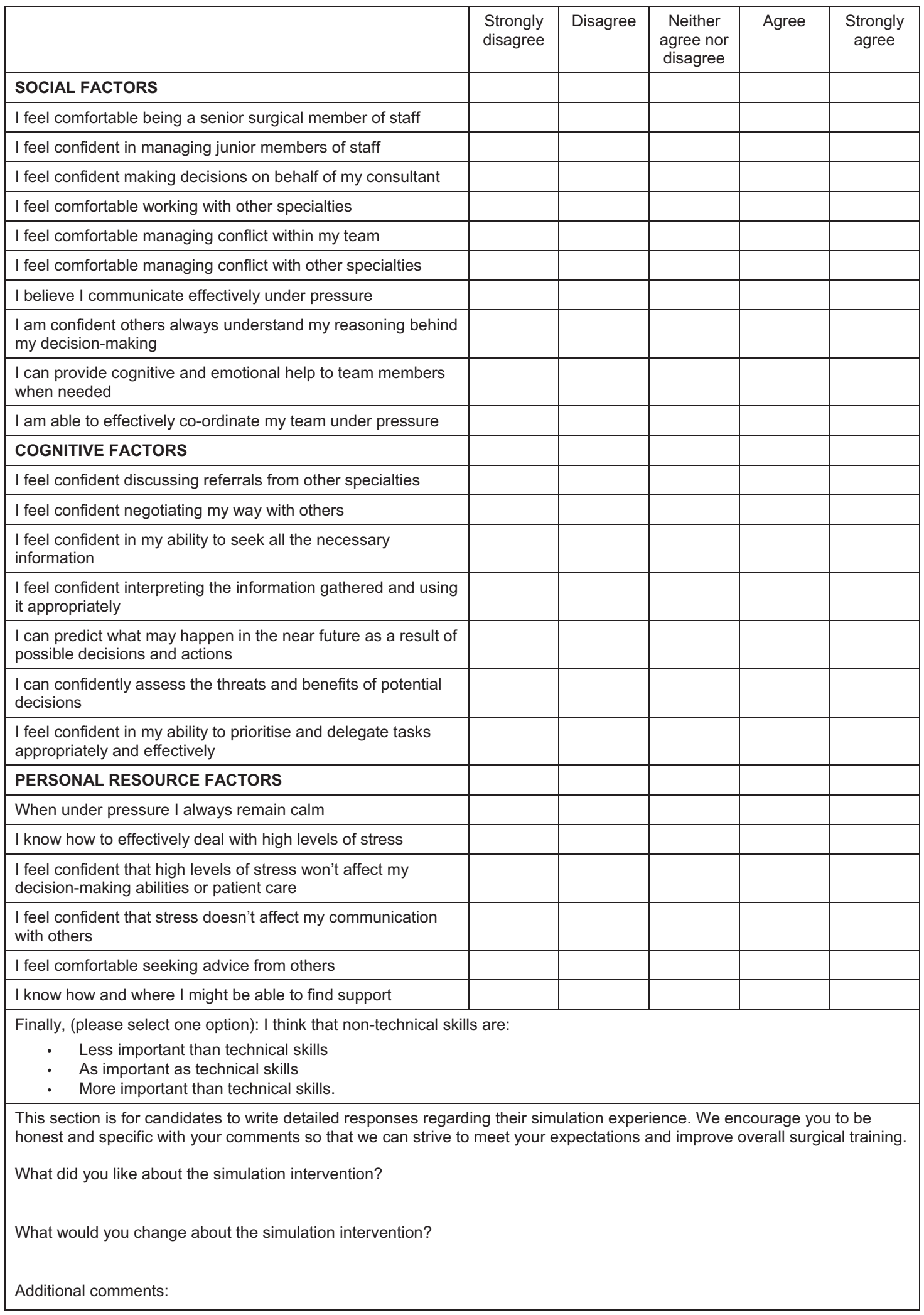

